\title{
The Future of Computational Asteroseismology
}

\author{
Travis S. Metcalfe \\ High Altitude Observatory \& Scientific Computing Division, \\ National Center for Atmospheric Research, P.O. Box 3000, Boulder CO 80307 USA
}

\begin{abstract}
The history of stellar seismology suggests that observation and theory often take turns advancing our understanding. The recent tripling of the sample of pulsating white dwarfs generated by the Sloan Digital Sky Survey represents a giant leap on the observational side. The time is ripe for a comparable advance on the theoretical side. There are basically two ways we can improve our theoretical understanding of pulsating stars: we can improve the fundamental ingredients of the models, or we can explore the existing models in greater computational detail. For pulsating white dwarfs, much progress has recently been made on both fronts: models now exist that connect the interior structure to its complete evolutionary history, while a method of using parallel computers for global exploration of relatively simple models has also been developed. Future advances in theoretical white dwarf asteroseismology will emerge by combining these two approaches, yielding unprecedented insight into the physics of diffusion, nuclear burning, and mixing.
\end{abstract}

\section{Context}

In just the past few years, the Sloan Digital Sky Survey has tripled the sample of pulsating white dwarf stars (Mukadam et al. 2004; Mullally et al. 2005; Kepler et al. 2005; Castanheira et al. 2006). In the next few years we can expect similar increases to emerge for other types of pulsating stars, from space missions such as CoRoT and Kepler. The observations are quickly becoming too numerous for us to do traditional model-fitting by hand. We need to automate the procedure so we can spend more of our time thinking about the results.

The great thing about computers is that they can work 24 hours a day, and they are so inexpensive that you can have many of them working for you at once (Metcalfe \& Nather 2000). There are many exciting areas of pulsating star research where analytical work can contribute much to our understanding (e.g. studies of the mode selection mechanism, intrinsic amplitude variability, and non-sinusoidal light variations, to name just a few), but I won't discuss them here. This will be a computer-centric view of the future. After a brief overview of white dwarf asteroseismology, I will outline two broad approaches that are currently being used to obtain physical insight from computational work. In the future, as our computing potential increases, we will eventually be able to combine these two approaches.

\section{White Dwarf Asteroseismology}

First let me remind you that there are several major spectroscopic classes of white dwarfs. With few exceptions, we expect all of them to have cores composed of a mixture of carbon and oxygen - the ashes of helium burning during the red giant phase (Metcalfe 2003). About $80 \%$ of white dwarfs are classified as type DA, with a mantle of helium above the core and beneath a thin surface layer of pure hydrogen. Most of the remaining $20 \%$ show no traces of hydrogen-exposing either a pure neutral (type DB) or ionized (type DO) helium surface. Each of these major spectral types produces its own class of variables in the H-R diagram, 
spanning the temperature ranges for partial ionization of carbon and oxygen (DOV), helium (DBV), and hydrogen (DAV). The instability strips for these three classes are roughly equally spaced in $\log T_{\text {eff }}$ (see Elsworth \& Thompson 2004, their Fig. 1).

Like other types of pulsating stars, the spherical symmetry allows us to model the variations with spherical harmonic functions, and because we do not have spatial resolution across the stellar surface only those modes with low spherical degree $(\ell \lesssim 3)$ can be detected. In contrast to other types of pulsating stars, the field of white dwarf asteroseismology developed early along with helioseismology. This can be attributed to the fact that the pulsations in these stars are not subtle. The total light variations are typically $\sim 10 \%$ on convenient timescales of $\sim 10$ minutes. The simultaneous presence of many closely-spaced frequencies leads to easily visible beating in their light curves.

From a theoretical perspective, pulsation frequencies are basically determined by the sound speed (or Lamb frequency) and the buoyancy (or Brunt-Väisälä) frequency from the centre of a star to its surface. Pressure modes ( $\mathrm{p}$-modes) are excited at relatively high frequencies (larger than both of these natural frequencies) while gravity modes (g-modes) are excited at lower frequencies, smaller than both natural frequencies (Unno et al. 1989). The pulsations in white dwarf stars are excited in the range of frequencies characteristic of g-modes, and the models suggest that the periods are determined primarily by the buoyancy frequency. The strong gravity in white dwarf stars quickly stratifies the surface layers, and the resulting composition gradients cause perturbations to the buoyancy frequency that lead to deviations from the uniform period spacing predicted by asymptotic theory in homogeneous models. We can use these deviations to infer the interior structure through forward modelling.

\section{Beyond Local Fitting}

One way that we can obtain physical insight from computational work is to use relatively simple models, but to explore them more globally than we have in the past. What does this mean in practice? Most of you are probably familiar with Moore's Law, which is really an empirical observation that "computing power per unit cost doubles approximately every 18 months." This has been true for more than a century, spanning many different computing technologies. A somewhat less well known law (because I made it up) is the More is Better Law, which states: "If you spend more time writing the paper than running the models, you didn't run enough models."

Of course, "writing the paper" is really just a euphemism for all aspects of a research project that do not involve either computing or interpreting the results. The point is that if you choose to take a computational approach to a problem, the actual computations should occupy a large fraction of the total time needed to complete the project. Thus, what we can accomplish is in some sense driven by Moore's Law - but we can circumvent this limitation with parallel computing, and then concentrate on what More we can do.

\section{More Stars}

Although our models are often physically simplified, we can instill greater confidence in the computational results by fitting them to more stars. If this leads to a qualitatively consistent picture of what we theoretically expect to find, it could just be a coincidence-but as more and more stars support the same picture, it is easier to believe that even relatively simple models can provide important physical insights. Let me give you an example from my own work on white dwarf stars.

The DB white dwarfs are thought to evolve from hydrogen-deficient post-asymptotic giant branch (post-AGB) stars, which are the result of a very late thermal pulse leading to the socalled born-again AGB scenario (Iben et al. 1983). The hot DO white dwarfs that emerge initially have envelopes containing a uniform mixture of helium, carbon, and oxygen. As the 

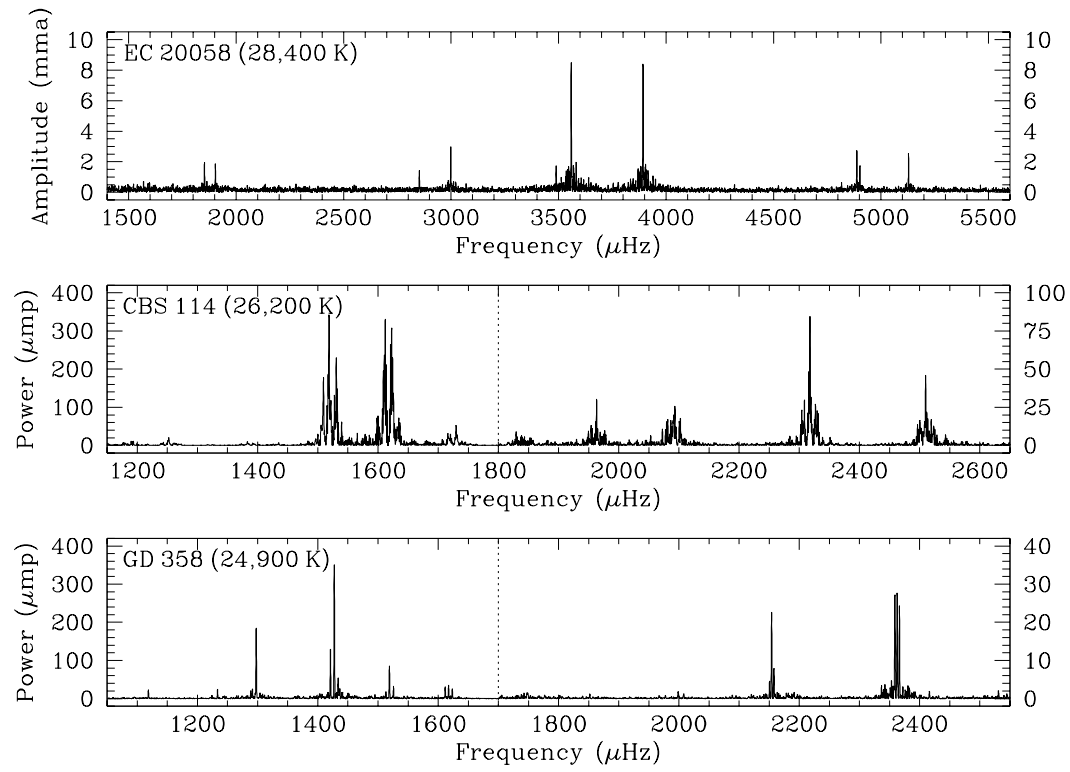

Figure 1: Fourier spectra from multi-site observations of three DBV white dwarfs with different temperatures. The data are from Sullivan et al. (in prep.), Metcalfe et al. (2005), and Winget et al. (1994); temperatures are from Beauchamp et al. (1999). Note that the top panel shows amplitude, while the middle and bottom panels show power with different scales for the left and right side of the vertical dotted line.

DO star cools over time, the helium floats to the surface-gradually growing thicker and transforming the star into a DB. This process continues within the DB instability strip, so we can test the theory by measuring the thickness of the pure helium surface layer in several DBV stars with different temperatures.

Asteroseismic observations are now available for three DBV stars which exhibit many independent pulsation modes (see Fig. 1). Adopting the spectroscopic temperatures of Beauchamp et al. (1999), the hottest of the stars is EC 20058 (Sullivan et al., in prep.) at $28400 \mathrm{~K}$. Slightly cooler is CBS 114 (Metcalfe et al. 2005) at $26200 \mathrm{~K}$, and cooler still is GD 358 (Winget et al. 1994) at $24900 \mathrm{~K}$. The specific prediction of diffusion theory is that we should find progressively thicker surface helium layers for cooler DB white dwarfs.

\section{More Models}

To test this prediction, we have used a parallel genetic algorithm (Metcalfe \& Charbonneau 2003 ) to globally minimize the root-mean-square (rms) difference between the observed and calculated pulsation periods in each of these three stars. We use relatively simple models with pure carbon cores since we are primarily interested in the envelope structure. The genetic algorithm searches a broad range for each of the four adjustable parameters, probing white dwarf masses $\left(M_{*}\right)$ between 0.45 and $0.95 \mathrm{M}_{\odot}$, effective temperatures ( $T_{\text {eff }}$ ) from 20000 to $30000 \mathrm{~K}$, total envelope masses $\left(M_{\text {env }}\right)$ between $10^{-2}$ and $10^{-4} M_{*}$, and surface helium layer masses $\left(M_{\mathrm{He}}\right)$ from $10^{-5}$ to $10^{-7} M_{*}$. For each model-fit, the genetic algorithm calculates more than 500000 models - initially distributed over the full range of parameter values, but ultimately concentrated near the region of the global solution. 
Table 1: Optimal model parameters for three DBV stars.

\begin{tabular}{lrrrl}
\hline Parameter & EC 20058 & CBS 114 & GD 358 & Uncertainty \\
\hline$T_{\text {eff }}(\mathrm{K}) \ldots \ldots$ & 28100 & 25800 & 23100 & \pm 100 \\
$M_{*}\left(\mathrm{M}_{\odot}\right) \ldots \ldots$ & 0.550 & 0.630 & 0.630 & \pm 0.005 \\
$\log \left(M_{\text {env }} / M_{*}\right) \ldots$ & -3.56 & -2.42 & -2.92 & \pm 0.02 \\
$\log \left(M_{\mathrm{He}} / M_{*}\right) \ldots$ & -6.42 & -5.96 & -5.90 & \pm 0.02 \\
$\operatorname{rms}(\mathrm{s}) \ldots \ldots \ldots$ & 1.89 & 2.33 & 2.26 & $\ldots$ \\
\hline
\end{tabular}

The results of the three model-fits (see Table 1) are in qualitative agreement with the predictions of diffusion theory, with the inferred surface helium layer masses growing thicker for the progressively cooler stars. The inferred masses and temperatures for the three stars are in reasonable agreement with the spectroscopically determined values, and the total envelope masses are all within the range expected from stellar evolution theory (D'Antona \& Mazzitelli 1979). The overall quality of each fit is quite good, especially considering that we have ignored any structure in the core.

\section{More Parameters}

Of course, we know that real white dwarfs do not have pure carbon cores. The actual interior chemical profiles consist of a uniform mixture of carbon and oxygen out to some fractional mass that depends on the size of the convective core in the red giant progenitor. Outside of this uniform region, the oxygen mass fraction decreases to zero in a manner that is determined by the conditions during helium shell burning. The most important feature of this chemical profile, from an asteroseismic standpoint, is the location of the initial break from a uniform mixture of carbon and oxygen. But the detailed shape of the oxygen mass fraction as it falls to zero also matters.

We can investigate the relative importance of these effects by adding more parameters to our model. In the past, inferences of core structure in white dwarfs were made using a simple parametrization of the chemical profile that fixed the oxygen mass fraction to its central value $\left(X_{0}\right)$ out to some fractional mass $(q)$ where it then decreased linearly to zero at the 0.95 fractional mass point (Metcalfe et al. 2001). Based on the calculations of Salaris et al. (1997), we have recently incorporated new chemical profiles into the models to specify the detailed shape of the oxygen mass fraction. We use the same two parameters, and simply scale the shape within each model.

The initial application of these 6-parameter models to CBS 114 yields a globally optimal model that agrees with both the predictions of diffusion theory and the expected nuclear burning history of the progenitor (Metcalfe 2005). This example clearly demonstrates the potential of using simple models when combined with a more global fitting strategy.

\section{Beyond the Spherical Cow}

At some point, even a global exploration of simple models will run into limitations. A broad search is certainly useful for identifying the region of the global solution, but the final results may suffer from small systematic errors in the optimal parameter values. How else might we use our continually expanding computational potential? Another approach is to build the best possible models, but limit the exploration. The analysis is local, but for a variety of reasons the final results may be More reliable. 


\section{More Physics}

The most obvious thing we can do to improve the models is simply to use the most accurate physical ingredients that are currently available. A recent example of this approach for white dwarf models can be found in Córsico et al. (2004). This study was designed to probe the asteroseismic differences between partially crystallized models of DAV stars with different core compositions. This is motivated by the fact that only relatively massive DA stars are expected to crystallize while still within the instability strip, and the transition from carbon and oxygen dominated cores to those containing primarily oxygen and neon takes place in this same mass range (Iben et al. 1997).

The authors include a time-dependent treatment of diffusion to describe the chemical profiles of the hydrogen and helium layers - making the calculations much more computationally demanding. They adopt initial profiles for the distribution of oxygen and neon in the cores of one set of models from detailed evolutionary calculations that follow the repeated carbonburning shell flashes in the progenitor. For the other set of models with carbon and oxygen cores, they include a self-consistent treatment of phase separation during the crystallization process. Although they make no attempt to fit these models to the available observations of BPM 37093 (Kanaan et al. 2005), they do find significant differences in the pulsation properties of the two sets of models which will ultimately make observational tests possible.

\section{More History}

We can also improve the white dwarf models by connecting them directly to the prior stages of their evolution. An impressive example of this approach was recently published by Althaus et al. (2005), who examine the possible evolutionary connection between DO, DB, and DQ stars. As outlined briefly before, the surface chemical composition of DO stars suggests that as they cool, diffusion will gradually transform them into DB white dwarfs. Since the surface convection zone grows deeper as the DB star cools further, it may eventually reach the underlying carbon-rich layer and dredge up enough carbon to transform the star again, this time into a DQ. Although there is no known class of DQ pulsators, asteroseismic tests of these evolutionary calculations are possible within both the DOV and DBV instability strips.

The study follows the complete evolution of a $2.7 \mathrm{M}_{\odot}$ star from the zero age main sequence, through mass loss on the AGB, and into the white dwarf regime. The authors employ a coupled treatment of nuclear burning and mixing, including five chemical time steps for each evolution step, which is especially important to follow the fast evolutionary phases like the born-again episode. They also adopt the double-diffusive mixing-length theory of Grossman \& Taam (1996) to allow non-instantaneous mixing for a fluid with composition gradients. While the paper does not include a pulsation analysis of any models, the asteroseismic results shown in Table 1 suggest that such work could be fruitful.

\section{More Dimensions}

All of the models we have been discussing so far are 1-D, so they assume spherical symmetry. While this is generally a very good assumption for white dwarf stars, we know that the effects of rotation and magnetic fields can break the spherical symmetry. It has recently become computationally feasible to perform star-in-a-box calculations (Turcotte et al. 2002), and some of the most important 3-D applications focus on core-collapse supernovae (Fryer et al. 2006), which are really just a type of white dwarf star hidden in the cocoon of its progenitor. So this is another way we might improve the models in the future.

One caveat that I should mention comes from my thesis adviser, Ed Nather. When asked about the difference between 1-D models and 3-D models, he replied: "3-D models are wrong in three dimensions." 


\section{The Future}

We have discussed two broad approaches to improving our understanding of pulsating stars using presently available computational resources. One option is to perform a global search by generating millions of simple models for comparison with the observations. Or, with similar resources, we can calculate a few complete evolutionary tracks using relatively sophisticated physical models. My prediction for the future should be uncontroversial: as computers get faster, they will eventually allow us to combine these two approaches and generate millions of complete evolutionary models, opening the door to new tests of fundamental physics in pulsating stars.

Acknowledgments. I would like to thank the meeting organizers for inviting me to give this review, and for permitting me to write it for a broader audience. The National Center for Atmospheric Research is a federally funded research and development center sponsored by the U.S. National Science Foundation.

\section{References}

Althaus L. G., Serenelli A. M., Panei J. A., et al., 2005, A\&A, 435, 631

Beauchamp A., Wesemael F., Bergeron P., et al., 1999, ApJ, 516, 887

Castanheira B. G., Kepler S. O., Mullally F., et al., 2006, A\&A, 450, 227

Córsico A. H., García-Berro E., Althaus L. G., Isern J., 2004, A\&A, 427, 923

D'Antona F., Mazzitelli I., 1979, A\&A, 74, 161

Elsworth Y. P., Thompson M. J., 2004, A\&G, 45, 14

Fryer C. L., Rockefeller G., Warren M. S., 2006, ApJ, 643, 292

Grossman S. A., Taam R. E., 1996, MNRAS, 283, 1165

Iben I. J., Kaler J. B., Truran J. W., Renzini A., 1983, ApJ, 264, 605

Iben I. J., Ritossa C., Garcia-Berro E., 1997, ApJ, 489, 772

Kanaan A., Nitta A., Winget D. E., et al., 2005, A\&A, 432, 219

Kepler S. O., Castanheira B. G., Saraiva M. F. O., et al., 2005, A\&A, 442, 629

Metcalfe T. S., 2003, ApJ, 587, L43

Metcalfe T. S., 2005, MNRAS, 363, L86

Metcalfe T. S., Charbonneau P., 2003, J. Computat. Phys., 185, 176

Metcalfe T. S., Nather R. E., 2000, Baltic Astron., 9, 479

Metcalfe T. S., Winget D. E., Charbonneau P., 2001, ApJ, 557, 1021

Metcalfe T. S., Nather R. E., Watson T. K., et al., 2005, A\&A, 435, 649

Mukadam A. S., Mullally F., Nather R. E., et al., 2004, ApJ, 607, 982

Mullally F., Thompson S. E., Castanheira B. G., et al., 2005, ApJ, 625, 966

Salaris M., Dominguez I., García-Berro E., et al., 1997, ApJ, 486, 413

Turcotte S., Bazan G., Castor J., et al., 2002, in Aerts C., Bedding T. R., Christensen-Dalsgaard J., eds, ASP Conf. Ser. Vol. 259, Radial and Nonradial Pulsations as Probes of Stellar Physics. Astron. Soc. Pac., San Francisco, p. 72

Unno W., Osaki Y., Ando H., Saio H., Shibahashi H., 1989, Nonradial oscillations of stars. University of Tokyo Press, Tokyo

Winget D. E., Nather R. E., Clemens J. C., et al., 1994, ApJ, 430, 839 


\section{DISCUSSION}

Christensen-Dalsgaard: I think I have to disagree with you. If you spend more time computing models than writing papers, you are probably not using enough time to interpret and present the results of the computations.

Metcalfe: You can do parallel processing, that is, you should be running the models for your next paper while writing the present one.

Christensen-Dalsgaard: I would like to discuss the excitation of oscillations in white dwarfs. Previously it was believed that the DAVs were excited by the opacity mechanism in the hydrogen-ionization zone. But later it was found that they were actually excited by convective driving, shown by the work of Brickhill.

Metcalfe: It's actually a cause-and-effect question. But I would like to refer that question to Mike Montgomery for a detailed answer.

Montgomery: The DAs have a significant convection zone that dominates energy transfer. Brickhill did discover that this contributes to the driving, but only by doing numerical simulations, not hydrodynamical, and this was the discovery as you put it. Yanqin Wu and Peter Goldreich have given that an analytical basis. I hesitate to use the words that this is "accepted" now, but it is the most promising explanation we have for driving in these objects. There may be some isolated cases where convection is not that important (such as a white dwarf which is accreting solar composition material), but for the majority of the objects this is probably the correct mechanism, for both DAVs and DBVs. I point out that the current generation of equilibrium models shows that convection should be important all across the instability strip, even at the blue edge.

Gough: Apropos of Joergen's first comment, permit me to mention a theorem which is so obvious that almost all funding agencies are oblivious to it: if more time is spent making observations with expensive equipment and reducing the data to palatable form than is devoted to analysing the results, then insufficient effort has been expended in extracting useful science from the valuable observations.

Bedding: Is it a coincidence or is it an odd that the instability strip of the DAs lies on the extension of the classical instability strip in the HR diagram?

Winget: Partial ionization and convection are coincident, i.e. partial ionization causes convection.

Quirion: We understand the blue edge well, but if you go towards the red edge, there is more and more convection, so we have something like a "generalized" $\kappa$-mechanism at work here.

Kepler: I think we are going into semantics. At the blue edge of the DAV instability strip there is no convection. Later, the convection zone is very thin and pulsations cannot be excited by convection. In the middle of the instability strip, where convection is important, it adds to the driving. At the blue edge, driving comes only from partial ionization, and therefore the amplitudes are smaller and the light curves are linear and sinusoidal. In the middle of the strip the amplitudes are higher, the light curves become nonlinear and we have many modes.

Christensen-Dalsgaard: I'm not a specialist on white dwarfs, but the physics is different depending on whether the heating is caused by opacity variations or by convective effects. You are right that hydrogen is the cause of all of these, and these mechanisms are both heat engines, but the physical mechanism is different.

Quirion: You have the DA and DB stars, and then there's the DO and PG 1159 stars. These are spectroscopic designations. The so called DOVs are all variable PG 1159 stars, and not DO variables. This is easily confused and I don't know why people are keeping these designations.

Metcalfe: So you are suggesting to drop the DOV designation?

Kawaler: Spectroscopically, the PG 1159 stars are DOZQ stars! 